\title{
First order normalization in the generalized photo gravitational non-planar restricted three body problems
}

\author{
Nirbhay Kumar Sinha \\ Assistant Professor, Deshbandhu College,University of Delhi, Delhi, India \\ E-mail: nirbhay.k.sinha@gmail.com
}

\begin{abstract}
In this paper, we normalised the second-order part of the Hamiltonian of the problem. The problem is generalised in the sense that fewer massive primary is supposed to be an oblate spheroid. By photogravitational we mean that both primaries are radiating. With the help of Mathematica, $\mathrm{H}_{2}$ is normalised to $\mathrm{H}_{2}=\alpha_{1} \beta_{1} \omega_{1}+\alpha_{2} \beta_{2} \omega_{2}$. The resulting motion is composed of elliptic motion with a short period $\left(2 \pi / \omega_{1}\right)$, completed by an oscillation along the $\zeta$-axis with a short period $\left(2 \pi / \omega_{2}\right)$.
\end{abstract}

Keywords:Normalisation; Photogravitational; Non-Planar; RTBP.

\section{Introduction}

Normal forms are a standard tool in Hamiltonian mechanics to study the dynamics in a neighbourhood of invariant objects. Usually, these normal forms are obtained as divergent series, but their asymptotic character is what makes them useful. From theoretical point of view, they provide nonlinear approximations to the dynamics in a neighbourhood of the invariant object, that follows to obtain information about the real solutions of the system by taking the normal form up to a suitable finite order. The idea behind normal forms is to construct a transformation of phase-space that brings a given system of differential equations into the simplest possible form upto a certain order of accuracy.

From a more practical point of view, normal forms can be used as a computational method to obtain very accurate approximations to the dynamics in a neighbourhood of the selected invariant object, by neglecting the remainder. They have been applied, for example, to compute invariant manifolds or invariant tori. For this, it is necessary to compute the explicit expression of the normal form and of the canonical transformation that put the Hamiltonian into this reduced form. This computational formulation has special interest in some celestial mechanics model. It can be used to approximate the dynamics of some real world problems.

In this paper the first order normalisation have been studied analytically. We have examined the effect of gravitational potential, oblateness effect and radiation in out of plane equilibrium points in photogravitational restricted three body problem.

Sharma, R. K and Subba Rao, P.V (1976) discussed the three dimensional restricted three body problem with oblateness. Sokol'skii, A. G (1978) gave the proof of the stability of Lagrangian solutions for a critical mass ratio. Poschel, J. (1982) described the concept of integrability on Cantor sets for Hamiltonian systems. Meyer, K. R and Schmidt, D. S (1986) discussed the stability of the Lagrange Triangular Point and a theorem of Arnold. Celleti, A and Giorgilli, A (1991) examined the stability of the Lagrangian points in the spatial RTBP. Ito, H (1992) discussed the integrability of Hamiltonian systems and Birkhoff normal forms in the simple resonance case. Benettin, G et. al (1998) described stability of $\mathrm{L}_{4}$ and $\mathrm{L}_{5}$ in the spatial RTBP. Jorba, A (2001) gave numerical computation of the normal behaviour of invariant curves of n-dimensional maps. Oberti, P and Vienne, A (2003) dealt with a new theory improving the convergence of the solution around $\mathrm{L}_{4}$ in RTBP. Duskos, C. N and Markellos, V. V (2006) described out of plane equilibrium points in the RTBP with oblateness. Kusvah, B. S et. al (2007) examined non-linear stability of $\mathrm{L}_{4}$ in photogravitational RTBP with P-R drag.

Hence, we thought to examine non-linear stability of $\mathrm{L}_{4}$ in out of plane photogravitational RTBP with one of the primaries as an oblate spheroid. We have given short introduction of the problem. For non-linear stability of $\mathrm{L}_{4}$ in our problem, we first normalise $\mathrm{H}_{2}$ in section 2. With the help of Mathematica, we have normalised $\mathrm{H}_{2}$ of our problem as

$\mathrm{H}_{2}=\alpha_{1} \beta_{1} \omega_{1}+\alpha_{2} \beta_{2} \omega_{2}$

The resulting motion is composed of elliptic motion in $\zeta \eta$-plane with a short period $\left(2 \pi / \omega_{1}\right)$, completed by an oscillation along the $\zeta$-axis with a short period $\left(2 \pi / \omega_{2}\right)$.

\section{Normalisation of $\mathbf{H}_{2}$}

The second order part of the Hamiltonian $\mathrm{H}_{2}$ of our problem is given by

$$
H_{2}=\frac{P_{1}^{2}+P_{2}^{2}}{2}+n\left(p_{1} * z-p_{2} * x\right)-e x^{2}-f * z^{2}-g x z
$$

We suppose that $J=\{\{0,0,1,0\},\{0,0,0,1\},\{-1,0,0,0\},\{0,-1,0,0\}\}$. The transpose $[\mathrm{J}]=-\mathrm{J}$ is true.

With the help of Mathematica, we proceed further for normalisation of $\mathrm{H}_{2}$.

HessHqp $=\left\{\left\{\mathrm{D}\left[\mathrm{H}_{2}, \mathrm{x}\right]\right\},\left\{\mathrm{D}\left[\mathrm{H}_{2}, \mathrm{z}\right]\right\},\left\{\mathrm{D}\left[\mathrm{H}_{2}, \mathrm{p}_{1}\right]\right\},\left\{\mathrm{D}\left[\mathrm{H}_{2}, \mathrm{p}_{2}\right]\right\}\right.$

$=\left\{\left\{-2 \mathrm{ex}-\mathrm{gz}-\mathrm{np}_{2}\right\},\left\{-\mathrm{gx}-2 \mathrm{fz}+\mathrm{np}_{1}\right\},\left\{\mathrm{nz}+\mathrm{p}_{1}\right\},\left\{-\mathrm{nx}+\mathrm{p}_{2}\right\}\right\}$

Matrix form is given by 


$$
\left(\begin{array}{l}
-2 e x-g z-n p_{2} \\
-g x-2 f z+n p_{1} \\
n z+p_{1} \\
-n x+p_{2}
\end{array}\right)
$$

HessH $=\{\{-2 \mathrm{e},-\mathrm{g}, 0,-\mathrm{n}\},\{-\mathrm{g},-2 \mathrm{f}, \mathrm{n}, 0\},\{0, \mathrm{n}, 1,0\},\{-\mathrm{n}, 0,0,1\}\}$

Matrix form is $=\left(\begin{array}{cccc}-2 e & -g & 0 & -n \\ -g & -2 f & n & 0 \\ 0 & n & 1 & 0 \\ -n & 0 & 0 & 1\end{array}\right)$

Let $\mathrm{M}=\mathrm{J}$. HessH

$=\{\{0, \mathrm{n}, 1,0\},\{-\mathrm{n}, 0,0,1\},\{2 \mathrm{e}, \mathrm{g}, 0, \mathrm{n}\},\{\mathrm{g}, 2 \mathrm{f},-\mathrm{n}, 0\}\}$

Matrix form $=\left(\begin{array}{cccc}0 & n & 1 & 0 \\ -n & 0 & 0 & 1 \\ 2 e & g & 0 & n \\ g & 2 f & -n & 0\end{array}\right)$

Characteristic Polynomial [M, $\lambda$ ]

$=4 \mathrm{ef}-\mathrm{g}^{2}+2 \mathrm{en}{ }^{2}+2 \mathrm{fn} n^{2}+\mathrm{n}^{4}-2 \mathrm{e} \lambda^{2}-2 \mathrm{f} \lambda^{2}+2 \mathrm{n}^{2} \lambda^{2}+\lambda^{4}$

Collecting $\lambda$, we have

$=4 \mathrm{ef}-\mathrm{g}^{2}+2 \mathrm{en} \mathrm{n}^{2}+2 \mathrm{fn^{2 }}+\mathrm{n}^{4}+\left(-2 \mathrm{e}-2 \mathrm{f}+2 \mathrm{n}^{2}\right) \lambda^{2}+\lambda^{4}$

We suppose $A=\{\{-\lambda, n\},\{-n,-\lambda\}\}$

$$
\mathrm{B}=\{\{2 \mathrm{e}, \mathrm{g}\},\{\mathrm{g}, 2 \mathrm{f}\}\}
$$

Matrix form $=\left(\begin{array}{cc}2 e & g \\ g & 2 f\end{array}\right)$

$\mathrm{M}=\{\{\mathrm{A}, \mathrm{ID}\},\{\mathrm{B}, \mathrm{A}\}\}$

$=\{\{\{\{-\lambda, n\},\{-n, \lambda\}\}, I D\},\{\{\{2 \mathrm{e}, \mathrm{g}\},\{\mathrm{g}, 2 \mathrm{f}\}\},\{\{-\lambda, \mathrm{n}\},\{-\mathrm{n}$, $\lambda\}\}\}\}$

Let $X=\left\{x_{1}, x_{2}\right\}$, and $Y=\left\{x_{3}, x_{4}\right\}$ are the eigen vectors of $M$,

then $\mathrm{M}\{\mathrm{X}, \mathrm{Y}\}=\{0,0\}$

i.e. $\quad \mathrm{AX}+\mathrm{Y}=0$ or $\mathrm{Y}=-\mathrm{AX}$

A. $A=\left\{\left\{-n^{2}+\lambda^{2},-2 n \lambda\right\},\left\{2 n \lambda,-n^{2}+\lambda^{2}\right\}\right\}$

and $\mathrm{BX}+\mathrm{AY}=0$ or $\mathrm{Y}=(\mathrm{B}-\mathrm{A})^{2} \mathrm{X}=0$

i.e. $(B-A . A) \cdot X$

$=\left\{\left(2 \mathrm{e}+\mathrm{n}^{2}-\lambda^{2}\right) \mathrm{x}_{1}+(\mathrm{g}+2 \mathrm{n} \lambda) \mathrm{x}_{2},(\mathrm{~g}-2 \mathrm{n} \lambda) \mathrm{x}_{1}+\left(2 \mathrm{f}+\mathrm{n}^{2}-\lambda^{2}\right) \mathrm{x}_{2}\right\}$

i.e.

$$
\frac{x_{1}}{(g+2 n \lambda)}=\frac{x_{2}}{-\left(2 e+n^{2}-\lambda^{2}\right)}
$$

From $\mathrm{Y}=-\mathrm{A} . \mathrm{X}$

$\left\{\lambda \mathrm{x}_{1}-\mathrm{nx}_{2}, \mathrm{nx}_{1}-\lambda \mathrm{x}_{2}\right\}=-\mathrm{A} \cdot\left\{(\mathrm{g}+2 \mathrm{n} \lambda),-\left(2 \mathrm{e}+\mathrm{n}^{2}-\lambda^{2}\right)\right\}$

$=\left\{\lambda(\mathrm{g}+2 \mathrm{n} \lambda)-\mathrm{n}\left(-2 \mathrm{e}-\mathrm{n}^{2}+\lambda^{2}\right), \mathrm{n}(\mathrm{g}+2 \mathrm{n} \lambda)+\lambda\left(-2 \mathrm{e}-\mathrm{n}^{2}+\lambda^{2}\right)\right\}$

$\mathrm{x}_{1}=(\mathrm{g}+2 \mathrm{n} \lambda), \mathrm{x}_{2}=-\left(2 \mathrm{e}+\mathrm{n}^{2}-\lambda^{2}\right)$

$\mathrm{x}_{3}=\lambda(\mathrm{g}+2 \mathrm{n} \lambda)-\mathrm{n}\left(-2 \mathrm{e}-\mathrm{n}^{2}+\lambda^{2}\right)$

$\mathrm{x}_{4}=\mathrm{n}(\mathrm{g}+2 \mathrm{n} \lambda)+\lambda\left(-2 \mathrm{e}-\mathrm{n}^{2}+\lambda^{2}\right)$

$\mathrm{x}_{1}=\mathrm{x}_{1} / . \lambda \rightarrow \mathrm{I} \omega=\mathrm{g}+2 \mathrm{in} \omega$

$\mathrm{x}_{2}=\mathrm{x}_{2 /} \cdot \lambda \rightarrow \mathrm{I} \omega=-2 \mathrm{e}-\mathrm{n}^{2}-\omega^{2}$

$\mathrm{x}_{3}=$ Expand $\left[\mathrm{x}_{3 / .} \lambda \rightarrow \mathrm{I} \omega\right]=2 \mathrm{en}+\mathrm{n}^{3}+\mathrm{ig} \omega-\mathrm{n} \omega^{2}$

$\mathrm{x}_{4}=\operatorname{Expand}\left[\mathrm{x}_{4 / \cdot} \lambda \rightarrow \mathrm{I} \omega\right]=\mathrm{gn}-2 \mathrm{ie} \omega+\mathrm{in}^{2} \omega-\mathrm{i} \omega^{3}$

$\mathrm{u}=\left\{\mathrm{g},-2 \mathrm{e}-\mathrm{n}^{2}-\omega^{2}, 2 \mathrm{en}+\mathrm{n}^{3}-\mathrm{n} \omega^{2}, \mathrm{gn}\right\}$

$\mathrm{v}=\left\{2 \mathrm{n} \omega, 0, \mathrm{~g} \omega,-2 \mathrm{e} \omega+\mathrm{n}^{2} \omega-\omega^{3}\right\}$

$\mathrm{u}_{1}=\left\{\mathrm{g},-2 \mathrm{e}-\mathrm{n}^{2}-\omega_{1}^{2}, 2 \mathrm{en}+\mathrm{n}^{3}-\mathrm{n} \omega_{1}^{2}, \mathrm{gn}\right\}$

$\mathrm{u}_{2}=\left\{\mathrm{g},-2 \mathrm{e}-\mathrm{n}^{2}-\omega_{2}^{2}, 2 \mathrm{en}+\mathrm{n}^{3}-\mathrm{n} \omega_{2}^{2}, \mathrm{gn}\right\}$

$\mathrm{v}_{1}=\left\{2 \mathrm{n} \omega_{1}, 0, \mathrm{~g} \omega_{1},-2 \mathrm{e} \omega_{1}+\mathrm{n}^{2} \omega_{1}-\omega_{1}^{3}\right\}$

$\mathrm{v}_{2}=\left\{2 \mathrm{n} \omega_{2}, 0, \mathrm{~g} \omega_{2},-2 \mathrm{e} \omega_{2}+\mathrm{n}^{2} \omega_{2}-\omega_{2}^{3}\right\}$

$\operatorname{ctmp}=\left\{\left\{\mathrm{g},-2 \mathrm{e}-\mathrm{n}^{2}-\omega_{1}^{2}, 2 \mathrm{en}+\mathrm{n}^{3}-\mathrm{n} \omega_{1}^{2}\right.\right.$, gn $\},\left\{\mathrm{g},-2 \mathrm{e}-\mathrm{n}^{2}-\omega_{2}^{2}, 2 \mathrm{en}+\mathrm{n}^{3}\right.$ - n $\omega_{2}^{2}$, gn $\}$,

$\left\{2 \mathrm{n} \omega_{1}, 0, \mathrm{~g} \omega_{1},-2 \mathrm{e} \omega_{1}+\mathrm{n}^{2} \omega_{1}-\omega_{1}^{3}\right\},\left\{2 \mathrm{n} \omega_{2}, 0, \mathrm{~g} \omega_{2},-\right.$

$\left.\left.2 \mathrm{e} \omega_{2}+\mathrm{n}^{2} \omega_{2}-\omega_{2}^{3}\right\}\right\}$

$\mathrm{c}=$ Transpose $[\mathrm{ctmp}]$

$=\left\{\left\{\mathrm{g}, \mathrm{g}, 2 \mathrm{n} \omega_{1}, 2 \mathrm{n} \omega_{2}\right\},\left\{-2 \mathrm{e}-\mathrm{n}^{2}-\omega_{1}^{2},-2 \mathrm{e}-\mathrm{n}^{2}-\omega_{2}^{2}, 0,0\right\}\right.$,

$\left\{2 \mathrm{en}+\mathrm{n}^{3}-\mathrm{n} \omega_{1}^{2}, 2 \mathrm{en}+\mathrm{n}^{3}-\mathrm{n} \omega_{2}^{2}, \mathrm{~g} \omega_{1}, \mathrm{~g} \omega_{2}\right\}$,

$\left\{\right.$ gn, gn, $\left.\left.-2 \mathrm{e} \omega_{1}+\mathrm{n}^{2} \omega_{1}-\omega_{1}^{3},-2 \mathrm{e} \omega_{2}+\mathrm{n}^{2} \omega_{2}-\omega_{2}^{3}\right\}\right\}$
Transpose [c] .J.c

$=\left\{\left\{\mathrm{g}\left(2 \mathrm{en}+\mathrm{n}^{3}-\mathrm{n} \omega_{1}^{2}\right)+\mathrm{g}\left(-2 \mathrm{en}-\mathrm{n}^{3}+\mathrm{n} \omega_{1}^{2}\right)\right.\right.$,

gn $\left(-2 e-n^{2}-\omega_{1}^{2}\right)+g\left(-2 e n-n^{3}+n \omega_{1}^{2}\right)-g n\left(-2 e-n^{2}-\omega_{2}^{2}\right)+g(2 e n$

$\left.+\mathrm{n}^{3}-\mathrm{n} \omega_{2}^{2}\right)$

$\mathrm{g}^{2} \omega_{1}+2 n \omega_{1}\left(-2 e n-n^{3}+n \omega_{1}^{2}\right)+\left(-2 e-n^{2}-\omega_{1}^{2}\right)\left(-2 e \omega_{1}+n^{2} \omega_{1}-\right.$

$\left.\omega_{1}^{3}\right)$,

$\mathrm{g}^{2} \omega_{2}+2 \mathrm{n} \omega_{2}\left(-2 e n-n^{3}+n \omega_{1}^{2}\right)+\left(-2 e-n^{2}-\omega_{1}^{2}\right)\left(-2 e \omega_{2}+n^{2} \omega_{2}-\right.$

$\left.\left.\omega_{2}^{3}\right)\right\}$,

$\left\{-\operatorname{gn}\left(-2 e-n^{2}-\omega_{1}^{2}\right)+g\left(2 e n+n^{3}-n \omega_{1}^{2}\right)+g n\left(-2 e-n^{2}-\omega_{2}^{2}\right)+g(-\right.$ $\left.2 \mathrm{en}-\mathrm{n}^{3}+\mathrm{n} \omega_{2}^{2}\right)$,

$\mathrm{g}\left(2 \mathrm{en}+\mathrm{n}^{3}-\mathrm{n} \omega_{2}^{2}\right)+\mathrm{g}\left(-2 \mathrm{en}-\mathrm{n}^{3}+\mathrm{n} \omega_{2}^{2}\right)$,

$\mathrm{g}^{2} \omega_{1}+\left(-2 \mathrm{e} \omega_{1}+\mathrm{n}^{2} \omega_{1}-\omega_{1}^{3}\right)\left(-2 \mathrm{e}-\mathrm{n}^{2}-\omega_{2}^{2}\right)+2 \mathrm{n} \omega_{1}\left(-2 e n-n^{3}+\mathrm{n}\right.$

$\left.\omega_{2}^{2}\right)$,

$\mathrm{g}^{2} \omega_{2}+2 \mathrm{n} \omega_{2}\left(-2 \mathrm{en}-\mathrm{n}^{3}+\mathrm{n} \omega_{2}^{2}\right)+\left(-2 \mathrm{e}-\mathrm{n}^{2}-\omega_{2}^{2}\right)\left(-2 \mathrm{e} \omega_{2}+\mathrm{n}^{2} \omega_{2}-\right.$

$\left.\left.\omega_{2}^{3}\right)\right\}$,

$\left\{-g^{2} \omega_{1}+2 n \omega_{1}\left(2 e n+n^{3}-n \omega_{1}^{2}\right)+\left(-2 e-n^{2}-\omega_{1}^{2}\right)\left(2 e \omega_{1}-n^{2} \omega_{1}+\right.\right.$ $\left.\omega_{1}^{3}\right)$,

$-\mathrm{g}^{2} \omega_{1}+\left(2 \mathrm{e} \omega_{1}-\mathrm{n}^{2} \omega_{1}+\omega_{1}^{3}\right)\left(-2 \mathrm{e}-\mathrm{n}^{2}-\omega_{2}^{2}\right)+2 \mathrm{n} \omega_{1}\left(2 \mathrm{en}+\mathrm{n}^{3}-\mathrm{n}\right.$ $\left.\left.\omega_{2}^{2}\right), 0,0\right\}$,

$\left\{-\mathrm{g}^{2} \omega_{2}+2 \mathrm{n} \omega_{2}\left(2 \mathrm{en}+\mathrm{n}^{3}-\mathrm{n} \omega_{1}^{2}\right)+\left(-2 \mathrm{e}-\mathrm{n}^{2}-\omega_{1}^{2}\right)\left(2 \mathrm{e} \omega_{2}-\mathrm{n}^{2} \omega_{2}+\right.\right.$ $\left.\omega_{2}^{3}\right)$,

$-\mathrm{g}^{2} \omega_{2}+2 n \omega_{2}\left(2 e n+n^{3}-n \omega_{2}^{2}\right)+\left(-2 e-n^{2}-\omega_{2}^{2}\right)\left(2 e \omega_{2}-n^{2} \omega_{2}+\right.$ $\left.\left.\left.\omega_{2}^{3}\right), 0,0\right\}\right\}$

Expand

$\left\{\left\{0,0,4 \mathrm{e}^{2} \omega_{1}+\mathrm{g}^{2} \omega_{1}-4 \mathrm{en}^{2} \omega_{1}-3 \mathrm{n}^{4} \omega_{1}+4 \mathrm{e} \omega_{1}^{3}+2 \mathrm{n}^{2} \omega_{1}^{3}+\omega_{1}^{5}\right.\right.$, $4 \mathrm{e}^{2} \omega_{2}+\mathrm{g}^{2} \omega_{2}-4 \mathrm{en}^{2} \omega_{2}-3 \mathrm{n}^{4} \omega_{2}+2 \mathrm{e} \omega_{1}^{2} \omega_{2}+\mathrm{n}^{2} \omega_{1}^{2} \omega_{2}+2 \mathrm{e} \omega_{2}^{3}+\mathrm{n}^{2}$ $\left.\omega_{2}^{3}+\omega_{1}^{2} \omega_{2}^{3}\right\}$,

$\left\{0,0,4 \mathrm{e}^{2} \omega_{1}+\mathrm{g}^{2} \omega_{1}-4 \mathrm{en}^{2} \omega_{1}-3 \mathrm{n}^{4} \omega_{1}+2 \mathrm{e} \omega_{1}^{3}+\mathrm{n}^{2} \omega_{1}^{3}+2 \mathrm{e} \omega_{1} \omega_{2}^{2}+\mathrm{n}^{2} \omega_{1}\right.$ $\omega_{2}^{2}+\omega_{1}^{3} \omega_{2}^{2}$,

$\left.4 \mathrm{e}^{2} \omega_{2}+\mathrm{g}^{2} \omega_{2}-4 \mathrm{en}^{2} \omega_{2}-3 \mathrm{n}^{4} \omega_{2}+4 \mathrm{e} \omega_{2}^{3}+2 \mathrm{n}^{2} \omega_{2}^{3}+\omega_{2}^{5}\right\}$,

$\left\{-4 \mathrm{e}^{2} \omega_{1}-\mathrm{g}^{2} \omega_{1}+4 \mathrm{en}^{2} \omega_{1}+3 \mathrm{n}^{4} \omega_{1}-4 \mathrm{e} \omega_{1}^{3}-2 \mathrm{n}^{2} \omega_{1}^{3}-\omega_{1}^{5}\right.$,

$-4 \mathrm{e}^{2} \omega_{1}-\mathrm{g}^{2} \omega_{1}+4 \mathrm{en}^{2} \omega_{1}+3 \mathrm{n}^{4} \omega_{1}-2 \mathrm{e} \omega_{1}^{3}-\mathrm{n}^{2} \omega_{1}^{3}-2 \mathrm{e} \omega_{1} \omega_{2}^{2}-\mathrm{n}^{2} \omega_{1} \omega_{2}^{2}-$

$\left.\omega_{1}^{3} \omega_{2}^{2}, 0,0,\right\}$,

$\left\{-4 e^{2} \omega_{2}-g^{2} \omega_{2}+4 e^{2} \omega_{2}+3 n^{4} \omega_{2}-2 e \omega_{1}^{2} \omega_{2}-n^{2} \omega_{1}^{2} \omega_{2}-2 e \omega_{2}^{3}-n^{2}\right.$ $\omega_{2}^{3}-\omega_{1}^{2} \omega_{2}^{3}$,

$\left\{-4 \mathrm{e}^{2} \omega_{2}-\mathrm{g}^{2} \omega_{2}+4 \mathrm{en}^{2} \omega_{2}+3 \mathrm{n}^{4} \omega_{2}-4 \mathrm{e} \omega_{2}^{3}-2 \mathrm{n}^{2} \omega_{2}^{3}-\omega_{2}^{5}, 0,0\right\}$

Simplify

$\left\{\left\{0,0, \omega_{1}\left(4 \mathrm{e}^{2} \omega_{1}+\mathrm{g}^{2}-4 \mathrm{en}^{2}-3 \mathrm{n}^{4}+2\left(2 \mathrm{e}+\mathrm{n}^{2}\right) \omega_{1}^{2}+\omega_{1}^{4}\right)\right.\right.$,

$\left.\omega_{2}\left(4 \mathrm{e}^{2}+\mathrm{g}^{2}-4 \mathrm{en}^{2}-3 \mathrm{n}^{4}+\left(2 \mathrm{e}+\mathrm{n}^{2}\right) \omega_{2}^{3}+\omega_{1}^{2}\left(2 \mathrm{e}+\mathrm{n}^{2}+\omega_{2}^{2}\right)\right)\right\}$,

$\left\{0,0, \omega_{1}\left(4 \mathrm{e}^{2}+\mathrm{g}^{2}-4 \mathrm{en}^{2}-3 \mathrm{n}^{4}+\left(2 \mathrm{e}+\mathrm{n}^{2}\right) \omega_{2}^{2}+\omega_{1}^{2}\left(2 \mathrm{e}+\mathrm{n}^{2}+\right.\right.\right.$

$\left.\left.\omega_{2}^{2}\right)\right)$

$\left.\omega_{2}\left(4 \mathrm{e}^{2}+\mathrm{g}^{2}-4 \mathrm{en}^{2}-3 \mathrm{n}^{4}+2\left(2 \mathrm{e}+\mathrm{n}^{2}\right) \omega_{2}^{2}+\omega_{2}^{4}\right)\right\}$,

$\left\{-\omega_{1}\left(4 \mathrm{e}^{2}+\mathrm{g}^{2}-4 \mathrm{en}^{2}-3 \mathrm{n}^{4}+2\left(2 \mathrm{e}+\mathrm{n}^{2}\right) \omega_{1}^{2}+\omega_{1}^{4}\right)\right.$,

$-\omega_{1}\left(4 \mathrm{e}^{2}+\mathrm{g}_{2}-4 \mathrm{en}^{2}-3 \mathrm{n}^{4}+\left(2 \mathrm{e}+\mathrm{n}^{2}\right) \omega_{2}^{2}+\omega_{1}^{2}\left(2 \mathrm{e}+\mathrm{n}^{2}+\omega_{2}^{2}\right)\right) 0$, $0\}$,

$\left\{-\omega_{2}\left(4 e^{2}+g^{2}-4 e n^{2}-3 n^{4}+\left(2 e+n^{2}\right) \omega_{2}^{2}+\omega_{1}^{2}\left(2 e+n^{2}+\omega_{2}^{2}\right)\right)\right.$, $\left.\left.-\omega_{2}\left(4 \mathrm{e}^{2}+\mathrm{g}^{2}-4 \mathrm{en}^{2}-3 \mathrm{n}^{4}+2\left(2 \mathrm{e}+\mathrm{n}^{2}\right) \omega_{2}^{2}+\omega_{2}^{4}\right), 0,0\right\}\right\}$

Using the followings

$4 \mathrm{ef}-\mathrm{g}^{2}+2 \mathrm{en}^{2}+2 \mathrm{f} \mathrm{n}^{2}+\mathrm{n}^{4}+\left(-2 \mathrm{e}-2 \mathrm{f}+2 \mathrm{n}^{2}\right) \lambda^{2}+\lambda^{4} / . \lambda \rightarrow \mathrm{I} \omega$

$4 e f-g^{2}+2 e n^{2}+2 f n^{2}+n^{4}-\left(-2 e-2 f+2 n^{2}\right) \omega^{2}+\omega^{4}$ 
$n^{4}=-\left(4 e f-g^{2}+2 e n^{2}+2 f n^{2}-\left(-2 e-2 f+2 n^{2}\right) \omega^{2}+\omega^{4}\right)$

$\omega_{1}^{2}+\omega_{2}^{2}=\left(-2 e-2 f+2 n^{2}\right),\left(\omega_{1}^{2} \omega_{2}^{2}\right)=4 e f-g^{2}+2 e n^{2}+2 f n^{2}+n^{4}$

$\left\{\left\{0,0, \omega_{1}\left(4 \mathrm{e}^{2}+\mathrm{g}^{2}-4 \mathrm{en}^{2}-3 \mathrm{n}^{4}+2\left(2 \mathrm{e}+\mathrm{n}^{2}\right) \omega_{1}^{2}+\omega_{1}^{4}\right), 0\right\}\right.$,

$\left\{0,0,0, \omega_{2}\left(4 \mathrm{e}^{2}+\mathrm{g}^{2}-4 \mathrm{en}^{2}-3 \mathrm{n}^{4}+2\left(2 \mathrm{e}+\mathrm{n}^{2}\right) \omega_{2}^{2}+\omega_{2}^{4}\right)\right\}$,

$\left\{-\omega_{1}\left(4 e^{2}+g^{2}-4 e n^{2}-3 n^{4}+2\left(2 e+n^{2}\right) \omega_{1}^{2}+\omega_{1}^{4}\right), 0,0,0\right\}$,

$\left.\left\{0,-\omega_{2}\left(4 e^{2}+g^{2}-4 e n^{2}-3 n^{4}+2\left(2 e+n^{2}\right) \omega_{2}^{2}+\omega_{2}^{4}\right), 0,0\right\}\right\}$

$\omega_{1}\left(4 \mathrm{e}^{2}+\mathrm{g}^{2}-4 \mathrm{en}^{2}+2\left(2 \mathrm{e}+\mathrm{n}^{2}\right) \omega_{1}^{2}+\omega_{1}^{4}+\right.$

$3\left(4 e f-g^{2}+2 e n^{2}+2 f n^{2}-\left(-2 e-2 f+2 n^{2}\right) \omega_{1}^{2}+\omega_{1}^{4}\right.$

Simplify

$2 \omega_{1}\left(2 e^{2}-g^{2}+3 f n^{2}+e\left(6 f+n^{2}\right)+\left(5 e+3 f-2 n^{2}\right) \omega_{1}^{2}+2 \omega_{1}^{4}\right.$

$d \omega_{1}=2 \omega_{1}\left(2 e^{2}-g^{2}+e f n^{2}+e\left(6 f+n^{2}\right)+\left(5 e+3 f-2 n^{2}\right) \omega_{1}^{2}+2\right.$

$\left.\omega_{1}^{4}\right)$

$=\left(2 e^{2}-g^{2}+e f n^{2}+e\left(6 f+n^{2}\right)+\left(5 e+3 f-2 n^{2}\right) \omega_{1}^{2}+2 \omega_{1}^{4}\right)$

$d \omega_{2}=2 \omega_{2}\left(2 e^{2}-g^{2}+e f n^{2}+e\left(6 f+n^{2}\right)+\left(5 e+3 f-2 n^{2}\right) \omega_{2}^{2}+2\right.$

$\left.\omega_{1}^{4}\right)$

$=2 \omega_{2}\left(2 e^{2}-g^{2}+e f n^{2}+e\left(6 f+n^{2}\right)+\left(5 e+3 f-2 n^{2}\right) \omega_{2}^{2}+2 \omega_{2}^{4}\right)$

Transpose $[c] . J . c=\{\{0, D\},\{-D, 0\}\}$ where $D=\left\{\left\{d \omega_{1}, 0\right\},\{0\right.$, $\left.\left.\mathrm{d} \omega_{2}\right\}\right\}$

Matrix form $[\{\{0, D\},\{-D, 0\}\}]$

$=\left(\begin{array}{cc}0 & D \\ -D & 0\end{array}\right)$

Matrix form $\left[\left\{\left\{d \omega_{1}, 0\right\},\left\{0, d \omega_{2}\right\}\right\}\right]$

$=\left(\begin{array}{cc}d \omega_{1} & 0 \\ 0 & d \omega_{2}\end{array}\right)$

$\mathrm{J}=\{\{0,0,1,0\},\{0,0,0,1\},\{-1,0,0,0\},\{0,-1,0,0\}\}$

$\mathrm{cd} 1=\left\{\left\{\mathrm{g}, \mathrm{g}, 2 \mathrm{n} \omega_{1}, 2 \mathrm{n} \omega_{2}\right\},\left\{-2 \mathrm{e}-\mathrm{n}^{2}-\omega_{1}^{2},-2 \mathrm{e}-\mathrm{n}^{2}-\omega_{2}^{2}, 0,0\right\}\right.$,

$\left\{2 e n+n^{3}-n \omega_{1}^{2}, 2 e n+n^{3}-n \omega_{2}^{2}, g \omega_{1}, g \omega_{2}\right\}$,

$\left\{\right.$ gn, gn, $\left.\left.-2 \mathrm{e} \omega_{1}+\mathrm{n}^{2} \omega_{1}-\omega_{1}^{3},-2 \mathrm{e} \omega_{2}+\mathrm{n}^{2} \omega_{2}-\omega_{2}^{3}\right\}\right\}$

$\operatorname{cinv}=$ Inverse $[\mathrm{c}]$

$=\left\{\left\{\left(\left(-2 \mathrm{e}-\mathrm{n}^{2}-\omega_{2}^{2}\right)\left(\mathrm{g} \omega_{1}^{3} \omega_{2}-\mathrm{g} \omega_{1} \omega_{2}^{3}\right)\right) /\left(\mathrm{g}^{2} \omega_{1}^{5} \omega_{2}-8 \mathrm{en}^{2} \omega_{1}^{5}\right.\right.\right.$ $\omega_{2}-4 n^{4} \omega_{1}^{5} \omega_{2}-$

$2 \mathrm{~g}^{2} \omega_{1}^{3} \omega_{2}^{3}+16 \mathrm{n}^{2} \omega_{1}^{3} \omega_{2}^{3}+8 \mathrm{n}^{4} \omega_{1}^{3} \omega_{2}^{3}+\mathrm{g}^{2} \omega_{1} \omega_{2}^{5}-8 \mathrm{en}^{2} \omega_{1} \omega_{2}^{5}$

$\left.-4 n^{4} \omega_{1} \omega_{2}^{5}\right)$,

$\left(-\mathrm{g}^{2} \omega_{1}^{3} \omega_{2}+4 \mathrm{en}^{2} \omega_{1}^{3} \omega_{2}+2 \mathrm{n}^{4} \omega_{1}^{3} \omega_{2}+\mathrm{g}^{2} \omega_{1} \omega_{2}^{3}-4 \mathrm{en}^{2} \omega_{1} \omega_{2}^{3}-\right.$

$2 n^{4} \omega_{1} \omega_{2}^{3}-$

$\left.2 n^{2} \omega_{1}^{3} \omega_{2}^{3}+2 n^{2} \omega_{1} \omega_{2}^{5}\right) /\left(g^{2} \omega_{1}^{5} \omega_{2}-8 \mathrm{en}^{2} \omega_{1}^{5} \omega_{2}-4 n^{4} \omega_{1}^{5} \omega_{2}-\right.$

$2 \mathrm{~g}^{2} \omega_{1}^{3} \omega_{2}^{3}+$
$16 \mathrm{en}^{2} \omega_{1}^{3} \omega_{2}^{3}+8 \mathrm{n}^{4} \omega_{1}^{3} \omega_{2}^{3}+\mathrm{g}^{2} \omega_{1} \omega_{2}^{5}-8 \mathrm{en}^{2} \omega_{1} \omega_{2}^{5}-4 \mathrm{n}^{4} \omega_{1}$ $\left.\omega_{2}^{5}\right)$

$-\left(\left(-2 \mathrm{e}-\mathrm{n}^{2}-\omega_{2}^{2}\right)\left(2 \mathrm{n} \omega_{1}^{3} \omega_{2}-2 \mathrm{n} \omega_{1} \omega_{2}^{3}\right)\right) /\left(\mathrm{g}^{2} \omega_{1}^{5} \omega_{2}-8 \mathrm{en}{ }^{2} \omega_{1}^{5} \omega_{2}\right.$ $-4 n^{4} \omega_{1}^{5} \omega_{2}-$

$2 \mathrm{~g}^{2} \omega_{1}^{3} \omega_{2}^{3}+16 \mathrm{en}^{2} \omega_{1}^{3} \omega_{2}^{3}+8 \mathrm{n}^{4} \omega_{1}^{3} \omega_{2}^{3}+\mathrm{g}^{2} \omega_{1} \omega_{2}^{5}-8 \mathrm{en}^{2} \omega_{1} \omega_{2}^{5}-$ $\left.\left.4 n^{4} \omega_{1} \omega_{2}^{5}\right), 0\right\}$,

$\left\{-\left(\left(-2 \mathrm{e}-\mathrm{n}^{2}-\omega_{1}^{2}\right)\left(\mathrm{g} \omega_{1}^{3} \omega_{2}-\mathrm{g} \omega_{1} \omega_{2}^{3}\right)\right) /\left(\mathrm{g}^{2} \omega_{1}^{5} \omega_{2}-8 \mathrm{en}^{2} \omega_{1}^{5} \omega_{2}-\right.\right.$ $4 n^{4} \omega_{1}^{5} \omega_{2}-$

$2 \mathrm{~g}^{2} \omega_{1}^{3} \omega_{2}^{3}+16 \mathrm{en}^{2} \omega_{1}^{3} \omega_{2}^{3}+8 \mathrm{n}^{4} \omega_{1}^{3} \omega_{2}^{3}+\mathrm{g}^{2} \omega_{1} \omega_{2}^{5}-8 \mathrm{en}^{2} \omega_{1}$ $\left.\omega_{2}^{5}-4 n^{4} \omega_{1} \omega_{2}^{5}\right)$

$\left(g^{2} \omega_{1}^{3} \omega_{2}-4 \mathrm{en}^{2} \omega_{1}^{3} \omega_{2}-2 \mathrm{n}^{4} \omega_{1}^{3} \omega_{2}+2 \mathrm{n}^{2} \omega_{1}^{5} \omega_{2}-\mathrm{g}^{2} \omega_{1} \omega_{2}^{3}+\right.$ $4 \mathrm{en}^{2} \omega_{1} \omega_{2}^{3}+$ $\left.2 n^{4} \omega_{1} \omega_{2}^{3}-2 n^{2} \omega_{1}^{3} \omega_{2}^{3}\right) /\left(g^{2} \omega_{1}^{5} \omega_{2}-8 \mathrm{en}^{2} \omega_{1}^{5} \omega_{2}-4 n^{4} \omega_{1}^{5} \omega_{2}-\right.$ $2 \mathrm{~g}^{2} \omega_{1}^{3} \omega_{2}^{3}+$ $16 \mathrm{en}^{2} \omega_{1}^{3} \omega_{2}^{3}+8 \mathrm{n}^{4} \omega_{1}^{3} \omega_{2}^{3}+\mathrm{g}^{2} \omega_{1} \omega_{2}^{5}-8 \mathrm{en}^{2} \omega_{1} \omega_{2}^{5}-4 \mathrm{n}^{4} \omega_{1}$ $\left.\omega_{2}^{5}\right)$

$\left(\left(-2 \mathrm{e}-\mathrm{n}^{2}-\omega_{1}^{2}\right)\left(2 \mathrm{n} \omega_{1}^{3} \omega_{2}-2 \mathrm{n} \omega_{1} \omega_{2}^{3}\right)\right) /\left(\mathrm{g}^{2} \omega_{1}^{5} \omega_{2}-8 \mathrm{en}^{2} \omega_{1}^{5} \omega_{2}-\right.$ $4 n^{4} \omega_{1}^{5} \omega_{2}-$

$2 \mathrm{~g}^{2} \omega_{1}^{3} \omega_{2}^{3}+16 \mathrm{en}^{2} \omega_{1}^{3} \omega_{2}^{3}+8 \mathrm{n}^{4} \omega_{1}^{3} \omega_{2}^{3}+\mathrm{g}^{2} \omega_{1} \omega_{2}^{5}-8 \mathrm{en}^{2} \omega_{1} \omega_{2}^{5}-$ $\left.\left.4 n^{4} \omega_{1} \omega_{2}^{5}\right), 0\right\}$,

$\left\{\left(8 \mathrm{e}^{2} n \omega_{1}^{2} \omega_{2}+g^{2} n \omega_{1}^{2} \omega_{2}-2 n^{5} \omega_{1}^{2} \omega_{2}-8 \mathrm{e}^{2} n \omega_{2}^{3}-\mathrm{g}^{2} \mathrm{n} \omega_{2}^{3}+2 \mathrm{n}^{5} \omega_{2}^{3}\right.\right.$ +4 en $\omega_{1}^{2} \omega_{2}^{3}+$

$2 n^{3} \omega_{1}^{2} \omega_{2}^{3}-4$ en $\left.\omega_{2}^{5}-2 n^{3} \omega_{2}^{5}\right) /\left(g^{2} \omega_{1}^{5} \omega_{2}-8 e^{2} \omega_{1}^{5} \omega_{2}-4 n^{4} \omega_{1}^{5} \omega_{2}\right.$ $-2 \mathrm{~g}^{2} \omega_{1}^{3} \omega_{2}^{3}+$

$16 \mathrm{en}^{2} \omega_{1}^{3} \omega_{2}^{3}+8 \mathrm{n}^{4} \omega_{1}^{3} \omega_{2}^{3}+\mathrm{g}^{2} \omega_{1} \omega_{2}^{5}-8 \mathrm{en}^{2} \omega_{1} \omega_{2}^{5}-4 \mathrm{n}^{4} \omega_{1}$ $\left.\omega_{2}^{5}\right)$,

$\left(2\right.$ egn $\omega_{1}^{2} \omega_{2}+\mathrm{g} \mathrm{n}^{3} \omega_{1}^{2} \omega_{2}-2$ egn $\omega_{2}^{3}-\mathrm{g} \mathrm{n}^{3} \omega_{2}^{3}+\mathrm{gn} \omega_{1}^{2} \omega_{2}^{3}-\mathrm{gn}$ $\left.\omega_{2}^{5}\right) /$

$\left(g^{2} \omega_{1}^{5} \omega_{2}-8 e^{2} \omega_{1}^{5} \omega_{2}-4 n^{4} \omega_{1}^{5} \omega_{2}-2 g^{2} \omega_{1}^{3} \omega_{2}^{3}+\right.$ $16 \mathrm{en}^{2} \omega_{1}^{3} \omega_{2}^{3}+8 \mathrm{n}^{4} \omega_{1}^{3} \omega_{2}^{3}+\mathrm{g}^{2} \omega_{1} \omega_{2}^{5}-8 \mathrm{en}^{2} \omega_{1} \omega_{2}^{5}-4 \mathrm{n}^{4} \omega_{1}$ $\left.\omega_{2}^{5}\right)$

$\left(-2 \mathrm{eg} \omega_{1}^{2} \omega_{2}-\mathrm{gn}^{2} \omega_{1}^{2} \omega_{2}+2 \mathrm{eg} \omega_{2}^{3}+\mathrm{gn}^{2} \omega_{2}^{3}-\mathrm{g} \omega_{1}^{2} \omega_{2}^{3}+\mathrm{g} \omega_{2}^{5}\right)$ I

$\left(g^{2} \omega_{1}^{5} \omega_{2}-8 e^{2} \omega_{1}^{5} \omega_{2}-4 n^{4} \omega_{1}^{5} \omega_{2}-2 g^{2} \omega_{1}^{3} \omega_{2}^{3}+\right.$ $16 \mathrm{en}^{2} \omega_{1}^{3} \omega_{2}^{3}+8 \mathrm{n}^{4} \omega_{1}^{3} \omega_{2}^{3}+\mathrm{g}^{2} \omega_{1} \omega_{2}^{5}-8 \mathrm{en}^{2} \omega_{1} \omega_{2}^{5}-4 \mathrm{n}^{4} \omega_{1}$ $\left.\omega_{2}^{5}\right)$ 
$\left(-g^{2} \omega_{1}^{2} \omega_{2}+8 e n^{2} \omega_{1}^{2} \omega_{2}+4 n^{4} \omega_{1}^{2} \omega_{2}+g^{2} \omega_{2}^{3}-8 e n^{2} \omega_{2}^{3}-4 n^{4}\right.$ $\left.\omega_{2}^{3}\right) /$

$\left(\mathrm{g}^{2} \omega_{1}^{5} \omega_{2}-8 \mathrm{en}^{2} \omega_{1}^{5} \omega_{2}-4 \mathrm{n}^{4} \omega_{1}^{5} \omega_{2}-2 \mathrm{~g}^{2} \omega_{1}^{3} \omega_{2}^{3}+\right.$

$16 \mathrm{en}^{2} \omega_{1}^{3} \omega_{2}^{3}+8 \mathrm{n}^{4} \omega_{1}^{3} \omega_{2}^{3}+\mathrm{g}^{2} \omega_{1} \omega_{2}^{5}-8 \mathrm{en}^{2} \omega_{1} \omega_{2}^{5}-4 \mathrm{n}^{4} \omega_{1}$ $\left.\left.\omega_{2}^{5}\right)\right\}$

$\left\{\left(-8 e^{2} n \omega_{1}^{3}-g^{2} n \omega_{1}^{3}+2 n^{5} \omega_{1}^{3}-4 e n \omega_{1}^{5}-2 n^{3} \omega_{1}^{5}+8 e^{2} n \omega_{1} \omega_{2}^{2}+\right.\right.$ $\mathrm{g}^{2} \mathrm{n} \omega_{1} \omega_{2}^{2}-$

$2 n^{5} \omega_{1} \omega_{2}^{2}+4$ en $\left.\omega_{1}^{3} \omega_{2}^{2}+2 n^{3} \omega_{1}^{3} \omega_{2}^{2}\right) /\left(g^{2} \omega_{1}^{5} \omega_{2}-8 e n^{2} \omega_{1}^{5} \omega_{2}-4 n^{4}\right.$ $\omega_{1}^{5} \omega_{2}$

$2 \mathrm{~g}^{2} \omega_{1}^{3} \omega_{2}^{3}+16 \mathrm{en}^{2} \omega_{1}^{3} \omega_{2}^{3}+8 \mathrm{n}^{4} \omega_{1}^{3} \omega_{2}^{3}+\mathrm{g}^{2} \omega_{1} \omega_{2}^{5}-8 \mathrm{en}^{2} \omega_{1}$ $\left.\omega_{2}^{5}-4 n^{4} \omega_{1} \omega_{2}^{5}\right)$,

$\left(-2\right.$ egn $\omega_{1}^{3}-\operatorname{gn}^{3} \omega_{1}^{3}-\operatorname{gn} \omega_{1}^{5}+2 \operatorname{egn} \omega_{1} \omega_{2}^{2}+\operatorname{gn}^{3} \omega_{1} \omega_{2}^{2}+\operatorname{gn} \omega_{1}^{3}$ $\left.\omega_{2}^{2}\right) /$

$\left(\mathrm{g}^{2} \omega_{1}^{5} \omega_{2}-8 \mathrm{en}^{2} \omega_{1}^{5} \omega_{2}-4 \mathrm{n}^{4} \omega_{1}^{5} \omega_{2}-2 \mathrm{~g}^{2} \omega_{1}^{3} \omega_{2}^{3}+\right.$ $16 \mathrm{en}^{2} \omega_{1}^{3} \omega_{2}^{3}+8 \mathrm{n}^{4} \omega_{1}^{3} \omega_{2}^{3}+\mathrm{g}^{2} \omega_{1} \omega_{2}^{5}-8 \operatorname{en}^{2} \omega_{1} \omega_{2}^{5}-4 n^{4} \omega_{1}$ $\left.\left.\omega_{2}^{5}\right)\right\}$

$\left(2 \operatorname{eg} \omega_{1}^{3}+\operatorname{gn}^{2} \omega_{1}^{3}+g \omega_{1}^{5}-2 \operatorname{eg} \omega_{1} \omega_{2}^{2}-g^{2} \omega_{1} \omega_{2}^{2}-g \omega_{1}^{3} \omega_{2}^{2}\right) /\left(g^{2} \omega_{1}^{5} \omega_{2}-\right.$ $\operatorname{sen}^{2} \omega_{1}^{3} \omega_{2}-$

$4 n^{4} \omega_{1}^{5} \omega_{2}-2 g^{2} \omega_{1}^{3} \quad \omega_{2}^{3}+16 e^{2} \omega_{1}^{3} \quad \omega_{2}^{3}+8 n^{4} \omega_{1}^{3} \quad \omega_{2}^{3}+g^{2} \omega_{1} \omega_{2}^{5}-8 e n^{2} \omega_{1}$ $\left.\omega_{2}^{5}-4 n^{4} \omega_{1} \omega_{2}^{5}\right)$

$\left(g^{2} \omega_{1}^{3}-8 e^{2} \omega_{1}^{3}-4 n^{4} \omega_{1}^{3}-g^{2} \omega_{1} \omega_{2}^{2}+8 e^{2} \omega_{1} \omega_{2}^{2}+4 n^{4} \omega_{1} \omega_{2}^{2}\right)$ /

$\left(g^{2} \omega_{1}^{5} \omega_{2}-8 e^{2} \omega_{1}^{5} \omega_{2}-4 n^{4} \omega_{1}^{5} \omega_{2}-2 g^{2} \omega_{1}^{3} \omega_{2}^{3}+16 e n^{2} \omega_{1}^{3} \omega_{2}^{3}\right.$

$+8 n^{4} \omega_{1}^{3} \omega_{2}^{3}+$

$\left.\left.\left.\mathrm{g}^{2} \omega_{1} \omega_{2}^{5}-8 \mathrm{en}^{2} \omega_{1} \omega_{2}^{5}-4 \mathrm{n}^{4} \omega_{1} \omega_{2}^{5}\right)\right\}\right\}$,

Simplify

TRCHESSH2C = cinv.M.c

$\left\{\left\{-\frac{g\left(2 e+n^{2}+\omega_{2}^{2}\right)}{\left(g^{2}-4\left(2 e n^{2}+n^{4}\right)\right)\left(\omega_{1}^{2}-\omega_{2}^{2}\right)}, \frac{-g^{2}+4 e n^{2}+2 n^{4}-2 n^{2} \omega_{2}^{2}}{\left(g^{2}-4\left(2 e n^{2}+n^{4}\right)\right)\left(\omega_{1}^{2}-\omega_{2}^{2}\right)}\right.\right.$,

$\left.\frac{2 n\left(2 e+n^{2}+\omega_{2}^{2}\right)}{\left(g^{2}-4\left(2 e n^{2}+n^{4}\right)\right)\left(\omega_{1}^{2}-\omega_{2}^{2}\right)}, 0\right\},\left\{\frac{g\left(2 e+n^{2}+\omega_{1}^{2}\right)}{\left(g^{2}-4\left(2 e n^{2}+n^{4}\right)\right)\left(\omega_{1}^{2}-\omega_{2}^{2}\right)}\right.$,

$\left.\frac{g^{2}-2\left(2 e n^{2}+n^{4}\right)+2 n^{2} \omega_{1}^{2}}{\left(g^{2}-4\left(2 e n^{2}+n^{4}\right)\right)\left(\omega_{1}^{2}-\omega_{2}^{2}\right)},-\frac{2 n\left(2 e+n^{2}+\omega_{1}^{2}\right)}{\left(g^{2}-4\left(2 e n^{2}+n^{4}\right)\right)\left(\omega_{1}^{2}-\omega_{2}^{2}\right)}, 0\right\}$

$\left\{\frac{n\left(8 e^{2}+g^{2}-2 n^{4}+2\left(2 e+n^{2}\right) \omega_{2}^{2}\right)}{\left(g^{2}-4\left(2 e n^{2}+n^{4}\right)\right) \omega_{1}\left(\omega_{1}^{2}-\omega_{2}^{2}\right)}, \frac{g n\left(2 e+n^{2}+\omega_{2}^{2}\right)}{\left(g^{2}-4\left(2 e n^{2}+n^{4}\right)\right) \omega_{1}\left(\omega_{1}^{2}-\omega_{2}^{2}\right)}\right.$, $\left.-\frac{g\left(2 e+n^{2}+\omega_{2}^{2}\right)}{\left(g^{2}-4\left(2 e n^{2}+n^{4}\right)\right) \omega_{1}\left(\omega_{1}^{2}-\omega_{2}^{2}\right)}, \frac{1}{-\omega_{1}^{3}+\omega_{1} \omega_{2}^{2}}\right\}$,

$\left\{\frac{n\left(-8 e^{2}-g^{2}+2 n^{4}-2\left(2 e+n^{2}\right) \omega_{1}^{2}\right)}{\left(g^{2}-4\left(2 e n^{2}+n^{4}\right)\right) \omega_{2}\left(\omega_{1}^{2}-\omega_{2}^{2}\right)}, \frac{g n\left(2 e+n^{2}+\omega_{1}^{2}\right)}{\left(g^{2}-4\left(2 e n^{2}+n^{4}\right)\right) \omega_{2}\left(\omega_{1}^{2}-\omega_{2}^{2}\right)}\right.$,

$\left.\left.\frac{g\left(2 e+n^{2}+\omega_{1}^{2}\right)}{\left(g^{2}-4\left(2 e n^{2}+n^{4}\right)\right) \omega_{2}\left(\omega_{1}^{2}-\omega_{2}^{2}\right)}, \frac{1}{\omega_{1}^{2} \omega_{2}-\omega_{2}^{3}}\right\}\right\}$.

$\{\{0, \mathrm{n}, 1,0\},\{-\mathrm{n}, 0,0,1\},\{2 \mathrm{e}, \mathrm{g}, 0, \mathrm{n}\},\{\mathrm{g}, 2 \mathrm{f},-\mathrm{n}, 0\}\}$.

$\left\{\left\{\mathrm{g}, \mathrm{g}, 2 \mathrm{n} \omega_{1}, 2 \mathrm{n} \omega_{2}\right\},\left\{-2 \mathrm{e}-\mathrm{n}^{2}-\omega_{1}^{2},-2 \mathrm{e}-\mathrm{n}^{2}-\omega_{2}^{2}, 0,0\right\}\right.$,

$\left\{2 e n+n^{3}-n \omega_{1}^{2}, 2 e n+n^{3}-n \omega_{2}^{2}, g \omega_{1}, g \omega_{2}\right\}$,

$\left\{\right.$ gn, gn, $\left.-2 \mathrm{e} \omega_{1}+\mathrm{n}^{2} \omega_{1}-\omega_{1}^{3},-2 \mathrm{e} \omega_{2}+\mathrm{n}^{2} \omega_{2}-\omega_{2}^{3}\right\}$

$=\left\{\left\{\left(\left(2 \mathrm{en}+\mathrm{n}^{3}-\mathrm{n} \omega_{1}^{2}\right)\left(-2 \mathrm{e}-\mathrm{n}^{2}-\omega_{2}^{2}\right)\left(\mathrm{g} \omega_{1}^{3} \omega^{2}-\mathrm{g} \omega_{1} \omega_{2}^{3}\right)\right) /\right.\right.$

$\left(g^{2} \omega_{1}^{5} \omega_{2}-8 \operatorname{en}^{2} \omega_{1}^{5} \omega_{2}-4 n^{4} \omega_{1}^{5} \omega_{2}-2 g^{2} \omega_{1}^{3} \omega_{2}^{3}+16 \operatorname{en}^{2} \omega_{1}^{3} \omega_{2}^{3}+\right.$ $\left.8 n^{4} \omega_{1}^{3} \omega_{2}^{3}+g^{2} \omega_{1} \omega_{2}^{5}-8 \mathrm{en}^{2} \omega_{1} \omega_{2}^{5}-4 n^{4} \omega_{1} \omega_{2}^{5}\right)+\left(-2 \mathrm{e}-\mathrm{n}^{2}-\right.$ $\left.\omega_{1}^{2}\right)$

$\left(\left(\mathrm{n}\left(-2 \mathrm{e}-\mathrm{n}^{2}-\omega_{2}^{2}\right)\left(\mathrm{g} \omega_{1}^{3} \omega_{2}-\mathrm{g} \omega_{1} \omega_{2}^{3}\right)\right) /\left(\mathrm{g}^{2} \omega_{1}^{5} \omega_{2}-8 \mathrm{en}^{2} \omega_{1}^{5} \omega_{2}-4 \mathrm{n}^{4}\right.\right.$ $\omega_{1}^{5} \omega_{2}$

$-2 \mathrm{~g}^{2} \omega_{1}^{3} \omega_{2}^{3}+16 \mathrm{en}^{2} \omega_{1}^{3} \omega_{2}^{3}+8 \mathrm{n}^{4} \omega_{1}^{3} \omega_{2}^{3}+\mathrm{g}^{2} \omega_{1} \omega_{2}^{5}-8 \mathrm{en}^{2} \omega_{1} \omega_{2}^{5}$ $\left.-4 n^{4} \omega_{1} \omega_{2}^{5}\right)-$

$\left(\mathrm{g}\left(-2 \mathrm{e}-\mathrm{n}^{2}-\omega_{2}^{2}\right)\left(2 \mathrm{n} \omega_{1}^{3} \omega_{2}-2 \mathrm{n} \omega_{1} \omega_{2}^{3}\right)\right) /\left(\mathrm{g}^{2} \omega_{1}^{5} \omega_{2}-8 \mathrm{en}^{2} \omega_{1}^{5} \omega_{2}-\right.$ $4 n^{4} \omega_{1}^{5} \omega_{2}$

$-2 \mathrm{~g}^{2} \omega_{1}^{3} \omega_{2}^{3}+16 \mathrm{en}^{2} \omega_{1}^{3} \omega_{2}^{3}+8 \mathrm{n}^{4} \omega_{1}^{3} \omega_{2}^{3}+\mathrm{g}^{2} \omega_{1} \omega_{2}^{5}-8 \mathrm{en}^{2} \omega_{1} \omega_{2}^{5}-$ $\left.\left.4 n^{4} \omega_{1} \omega_{2}^{5}\right)\right)+$

gn $\left(-\left(\mathrm{n}\left(-2 \mathrm{e}-\mathrm{n}^{2}-\omega_{2}^{2}\right)\left(2 \mathrm{n} \omega_{1}^{3} \omega_{2}-2 \mathrm{n} \omega_{1} \omega_{2}^{3}\right)\right) /\left(\mathrm{g}^{2} \omega_{1}^{5} \omega_{2}-8 \mathrm{en}{ }^{2} \omega_{1}^{5}\right.\right.$ $\omega_{2}-4 n^{4} \omega_{1}^{5} \omega_{2}$

$-2 \mathrm{~g}^{2} \omega_{1}^{3} \omega_{2}^{3}+16 \mathrm{en}^{2} \omega_{1}^{3} \omega_{2}^{3}+8 \mathrm{n}^{4} \omega_{1}^{3} \omega_{2}^{3}+\mathrm{g}^{2} \omega_{1} \omega_{2}^{5}-8 \operatorname{en}^{2} \omega_{1} \omega_{2}^{5}$ $\left.-4 n^{4} \omega_{1} \omega_{2}^{5}\right)+$

$\left(-\mathrm{g}^{2} \omega_{1}^{3} \omega_{2}+4 \mathrm{en}^{2} \omega_{1}^{5} \omega_{2}+2 \mathrm{n}^{4} \omega_{1}^{3} \omega_{2}+\mathrm{g}^{2} \omega_{1} \omega_{2}^{3}-4 \mathrm{en}^{2} \omega_{1} \omega_{2}^{3}-2 \mathrm{n}^{4}\right.$ $\omega_{1} \omega_{2}^{3}-$

$\left.2 \mathrm{n}^{2} \omega_{1}^{3} \omega_{2}^{3}+2 \mathrm{n}^{2} \omega_{1} \omega_{2}^{5}\right) /\left(\mathrm{g}^{2} \omega_{1}^{5} \omega_{2}-8 \mathrm{en}^{2} \omega_{1}^{5} \omega_{2}-4 \mathrm{n}^{4} \omega_{1}^{5} \omega_{2}-\right.$ $2 \mathrm{~g}^{2} \omega_{1}^{3} \quad \omega_{2}^{3}+$ $16 \mathrm{en}^{2} \omega_{1}^{3} \omega_{2}^{3}+8 \mathrm{n}^{4} \omega_{1}^{3} \omega_{2}^{3}+\mathrm{g}^{2} \omega_{1} \omega_{2}^{5}-8 \mathrm{en}^{2} \omega_{1} \omega_{2}^{5}-4 n^{4} \omega_{1}$ $\left.\left.\omega_{1}^{5}\right)\right)+$ $\mathrm{g}\left(-\left(2 \mathrm{e}\left(-2 \mathrm{e}-\mathrm{n}^{2}-\omega_{2}^{3}\right)\left(2 \mathrm{n} \omega_{1}^{3} \omega_{2}-2 \mathrm{n} \omega_{1} \omega_{2}^{3}\right)\right) /\left(\mathrm{g}^{2} \omega_{1}^{5} \omega_{2}-8 \mathrm{en}^{2} \omega_{1}^{5}\right.\right.$ $\omega_{2}-4 n^{4} \omega_{1}^{5} \omega_{2}$

$-2 \mathrm{~g}^{2} \omega_{1}^{3} \omega_{2}^{3}+16 \mathrm{en}^{2} \omega_{1}^{3} \omega_{2}^{3}+8 \mathrm{n}^{4} \omega_{1}^{3} \omega_{2}^{3}+\mathrm{g}^{2} \omega_{1} \omega_{2}^{5}-8 \mathrm{en}^{2} \omega_{1} \omega_{2}^{5}$ $\left.-4 n^{4} \omega_{1} \omega_{2}^{5}\right)-$ 
$\left(\mathrm{n}\left(-\mathrm{g}^{2} \omega_{1}^{3} \omega_{2}+4 \mathrm{en}^{2} \omega_{1}^{3} \omega_{2}^{3} \omega_{2}+2 \mathrm{n}^{4} \omega_{1}^{3} \omega_{2}+\mathrm{g}^{2} \omega_{1} \omega_{2}^{3}-4 \mathrm{en}^{2} \omega_{1} \omega_{2}^{3}\right.\right.$ $-2 n^{4} \omega_{1} \omega_{2}^{3}-$

$\left.\left.2 \mathrm{n}^{2} \omega_{1}^{3} \omega_{2}^{3}+2 \mathrm{n}^{2} \omega_{1} \omega_{2}^{5}\right)\right) /\left(\mathrm{g}^{2} \omega_{1}^{5} \omega_{2}-8 \mathrm{en}^{2} \omega_{1}^{5} \omega_{2}-4 \mathrm{n}^{4} \omega_{1}^{5} \omega_{2}\right.$

$2 \mathrm{~g}^{2} \omega_{1}^{3} \omega_{2}^{3}+16 \mathrm{en}^{2} \omega_{1}^{3} \omega_{2}^{3}+8 \mathrm{n}^{4} \omega_{1}^{3} \omega_{2}^{3}+\mathrm{g}^{2} \omega_{1} \omega_{2}^{5}-8 \mathrm{en}^{2} \omega_{1} \omega_{2}^{5}$ $\left.\left.-4 n^{4} \omega_{1} \omega_{2}^{5}\right)\right)$

$\left(\left(-2 \mathrm{e}-\mathrm{n}^{2}-\omega_{2}^{2}\right)\left(2 \mathrm{en}+\mathrm{n}^{3}-\mathrm{n} \omega_{2}^{2}\right)\left(\mathrm{g} \omega_{1}^{3} \omega_{2}-\mathrm{g} \omega_{1} \omega_{2}^{3}\right)\right) /$

$\left(\mathrm{g}^{2} \omega_{1}^{5} \omega_{2}-8 \mathrm{en}^{2} \omega_{1}^{5} \omega_{2}-4 \mathrm{n}^{4} \omega_{1}^{5} \omega_{2}-2 \mathrm{~g}^{2} \omega_{1}^{3} \omega_{2}^{3}+16 \mathrm{en}^{2} \omega_{1}^{3} \omega_{2}^{3}\right.$

$+$

$\left.8 \mathrm{n}^{4} \omega_{1}^{3} \omega_{2}^{3}+\mathrm{g}^{2} \omega_{1} \omega_{2}^{5}-8 \mathrm{en}^{2} \omega_{1} \omega_{2}^{5}-4 \mathrm{n}^{4} \omega_{1} \omega_{2}^{5}\right)+\left(-2 \mathrm{e}-\mathrm{n}^{2}-\right.$

$\left.\omega_{2}^{2}\right)$

$\left(\left(\mathrm{n}\left(-2 \mathrm{e}-\mathrm{n}^{2}-\omega_{2}^{2}\right)\left(\mathrm{g} \omega_{1}^{3} \omega_{2}-\mathrm{g} \omega_{1} \omega_{2}^{3}\right)\right) /\left(\mathrm{g}^{2} \omega_{1}^{5} \omega_{2}-8 \mathrm{en}^{2} \omega_{1}^{5} \omega_{2}-\right.\right.$ $4 n^{4} \omega_{1}^{5} \omega_{2}-$

$2 \mathrm{~g}^{2} \omega_{1}^{3} \omega_{2}^{3}+16 \mathrm{en}^{2} \omega_{1}^{3} \omega_{2}^{3}+8 \mathrm{n}^{4} \omega_{1}^{3} \omega_{2}^{3}+\mathrm{g}^{2} \omega_{1} \omega_{2}^{5}-8 \mathrm{en}^{2} \omega_{1} \omega_{2}^{5}$ $\left.-4 n^{4} \omega_{1} \omega_{2}^{5}\right)-$

$\left(\mathrm{g}\left(-2 \mathrm{e}-\mathrm{n}^{2}-\omega_{2}^{2}\right)\left(2 \mathrm{n} \omega_{1}^{3} \omega_{2}-2 \mathrm{n} \omega_{1} \omega_{2}^{3}\right)\right) /\left(\mathrm{g}^{2} \omega_{1}^{5} \omega_{2}-8 \mathrm{en}^{2} \omega_{1}^{5} \omega_{2}\right.$ $-4 n^{4} \omega_{1}^{5} \omega_{2}$

$-2 \mathrm{~g}^{2} \omega_{1}^{3} \omega_{2}^{3}+16 \mathrm{en}^{2} \omega_{1}^{3} \omega_{2}^{3}+8 \mathrm{n}^{4} \omega_{1}^{3} \omega_{2}^{3}+\mathrm{g}^{2} \omega_{1} \omega_{2}^{5}-8 \mathrm{en}^{2} \omega_{1} \omega_{2}^{5}$

$\left.\left.-4 n^{4} \omega_{1} \omega_{2}^{5}\right)\right)+$

$\left(-2\right.$ egn $\omega_{1}^{3}-$ gn $^{3} \omega_{1}^{3}-$ gn $\omega_{1}^{5}+2 \operatorname{egn} \omega_{1} \omega_{2}^{2}+\operatorname{gn}^{3} \omega_{1} \omega_{2}^{2}+$ gn $\omega_{1}^{3}$ $\left.\omega_{2}^{2}\right) /$

$\left(\mathrm{g}^{2} \omega_{1}^{5} \omega_{2}-8 \mathrm{en}^{2} \omega_{1}^{5} \omega_{2}-4 \mathrm{n}^{4} \omega_{1}^{5} \omega_{2}-2 \mathrm{~g}^{2} \omega_{1}^{3} \omega_{2}^{3}+16 \mathrm{en}^{2} \omega_{1}^{3} \omega_{2}^{3}\right.$ $+8 n^{4} \omega_{1}^{3} \omega_{2}^{3}+$

$\left.\left.\mathrm{g}^{2} \omega_{1} \omega_{2}^{5}-8 \mathrm{en}^{2} \omega_{1} \omega_{2}^{5}-4 \mathrm{n}^{4} \omega_{1} \omega_{2}^{5}\right)\right)+2 \mathrm{n} \omega_{2}\left(\left(\mathrm{~g}\left(\mathrm{~g}^{2} \omega_{1}^{3}-8 \mathrm{en}{ }^{2} \omega_{1}^{3}\right.\right.\right.$ $-4 n^{4} \omega_{1}^{3}-$

$\left.\left.\mathrm{g}^{2} \omega_{1} \omega_{2}^{2}+8 \mathrm{en}^{2} \omega_{1} \omega_{2}^{2}+4 \mathrm{n}^{4} \omega_{1} \omega_{2}^{2}\right)\right) /\left(\mathrm{g}^{2} \omega_{1}^{5} \omega_{2}-8 \mathrm{en}^{2} \omega_{1}^{5} \omega_{2}-\right.$ $4 n^{4} \omega_{1}^{5} \omega_{2}$

$-2 \mathrm{~g}^{2} \omega_{1}^{3} \omega_{2}^{3}+16 \mathrm{en}^{2} \omega_{1}^{3} \omega_{2}^{3}+8 \mathrm{n}^{4} \omega_{1}^{3} \omega_{2}^{3}+\mathrm{g}^{2} \omega_{1} \omega_{2}^{5}-8 \mathrm{en}^{2} \omega_{1} \omega_{2}^{5}$

$\left.-4 n^{4} \omega_{1} \omega_{2}^{5}\right)+$

$\left(2 \mathrm{e}\left(2 \mathrm{eg} \omega_{1}^{3}+\mathrm{gn}^{2} \omega_{1}^{3}+\mathrm{g} \omega_{1}^{5}-2 \mathrm{eg} \omega_{1} \omega_{2}^{2}-\mathrm{gn}^{2} \omega_{1} \omega_{2}^{2}-\mathrm{g} \omega_{1}^{3}\right.\right.$ $\left.\omega_{2}^{2}\right) /$

$\left(g^{2} \omega_{1}^{5} \omega_{2}-8 \operatorname{en}^{2} \omega_{1}^{5} \omega_{2}-4 n^{4} \omega_{1}^{5} \omega_{2}-2 g^{2} \omega_{1}^{3} \omega_{2}^{3}+16 e n^{2} \omega_{1}^{3} \omega_{2}^{3}\right.$ $+$

$\left.8 \mathrm{n}^{4} \omega_{1}^{3} \omega_{2}^{3}+\mathrm{g}^{2} \omega_{1} \omega_{2}^{5}-8 \mathrm{en}^{2} \omega_{1} \omega_{2}^{5}-4 \mathrm{n}^{4} \omega_{1} \omega_{2}^{5}\right)-$

$\left(\mathrm{n}\left(-2 \mathrm{egn} \omega_{1}^{3}-\mathrm{gn}^{3} \omega_{1}^{3}-\mathrm{gn} \omega_{1}^{5}+2 \operatorname{egn} \omega_{1} \omega_{2}^{2}+\mathrm{gn}^{3} \omega_{1} \omega_{2}^{2}+\mathrm{gn}\right.\right.$ $\left.\left.\omega_{1}^{3} \omega_{2}^{2}\right)\right) /$ $\left(g^{2} \omega_{1}^{5} \omega_{2}-8 e^{2} \omega_{1}^{5} \omega_{2}-4 n^{4} \omega_{1}^{5} \omega_{2}-2 g^{2} \omega_{1}^{3} \omega_{2}^{3}+16 e n^{2} \omega_{1}^{3} \omega_{2}^{3}\right.$ $+8 n^{4} \omega_{1}^{3} \omega_{2}^{3}+$

$\left.\mathrm{g}^{2} \omega_{1} \omega_{2}^{5}-8 \mathrm{en}^{2} \omega_{1} \omega_{2}^{5}-4 \mathrm{n}^{4} \omega_{1} \omega_{2}^{5}\right)+\mathrm{g} \omega_{2}\left(-\left(\mathrm{n}\left(\mathrm{g}^{2} \omega_{1}^{3}-8 \mathrm{en}{ }^{2} \omega_{1}^{3}\right.\right.\right.$ $-4 n_{4} \omega_{1}^{3}-$

$\left.\left.\mathrm{g}^{2} \omega_{1} \omega_{2}^{2}+8 \mathrm{en}^{2} \omega_{1} \omega_{2}^{2}+4 \mathrm{n}^{4} \omega_{1} \omega_{2}^{2}\right)\right) /\left(\mathrm{g}^{2} \omega_{1}^{5} \omega_{2}-8 \mathrm{en}^{2} \omega_{1}^{5} \omega_{2}-\right.$ $4 n^{4} \omega_{1}^{5} \omega_{2}$

$-2 \mathrm{~g}^{2} \omega_{1}^{3} \omega_{2}^{3}+16 \mathrm{en}^{2} \omega_{1}^{3} \omega_{2}^{3}+8 \mathrm{n}^{4} \omega_{1}^{3} \omega_{2}^{3}+\mathrm{g}^{2} \omega_{1} \omega_{2}^{5}-8 \mathrm{en}^{2} \omega_{1} \omega_{2}^{5}$ $\left.-4 n^{4} \omega_{1} \omega_{2}^{5}\right)+$

$\left(-8 e^{2} n \omega_{1}^{3}-g^{2} n \omega_{1}^{3}+2 n^{5} \omega_{1}^{3}-4 e n \omega_{1}^{5}-2 n^{3} \omega_{1}^{5}+8 e^{2} n \omega_{1} \omega_{2}^{2}+\right.$ $\mathrm{g}^{2} \mathrm{n} \omega_{1} \omega_{2}^{2}-$

$2 n^{5} \omega_{1} \omega_{2}^{2}+4$ en $\left.\omega_{1}^{3} \omega_{2}^{2}+2 n^{3} \omega_{1}^{3} \omega_{2}^{2}\right) /\left(g^{2} \omega_{1}^{5} \omega_{2}-8 e^{2} \omega_{1}^{5} \omega_{2}-\right.$ $4 n^{4} \omega_{1}^{5} \omega_{2}-$

$2 \mathrm{~g}^{2} \omega_{1}^{3} \omega_{2}^{3}+16 \mathrm{en}^{2} \omega_{1}^{3} \omega_{2}^{3}+8 \mathrm{n}^{4} \omega_{1}^{3} \omega_{2}^{3}+\mathrm{g}^{2} \omega_{1} \omega_{2}^{5}-8 \mathrm{en}^{2} \omega_{1} \omega_{2}^{5}$ $\left.\left.\left.\left.-4 n^{4} \omega_{1} \omega_{2}^{5}\right)\right)\right\}\right\}$

Simplify

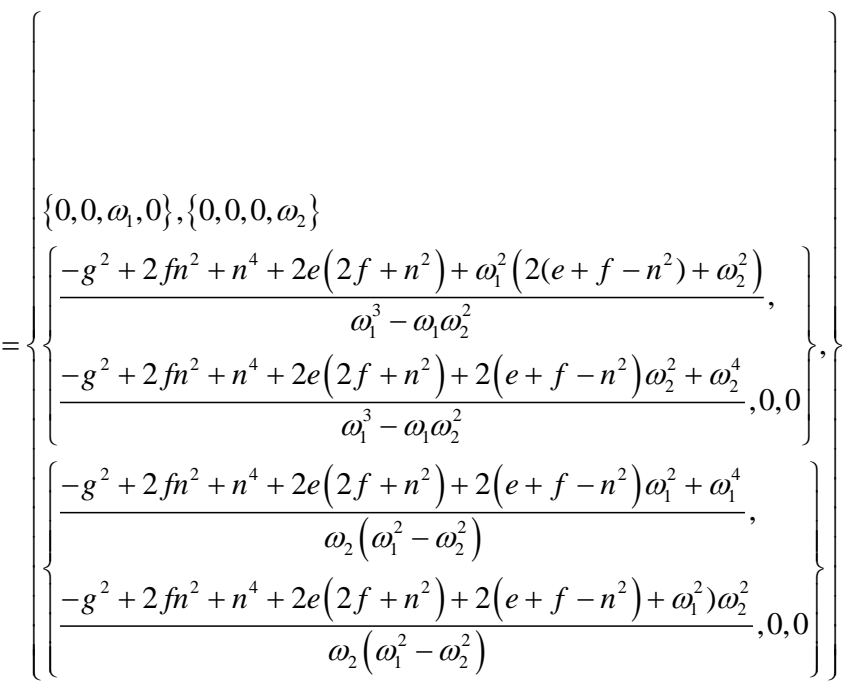

$\mathrm{d} \omega_{1}=2 \omega_{1}$

$\left(2 e^{2}-g^{2}+3 f n^{2}+e\left(6 f+n^{2}\right)+\left(5 e+3 f-2 n^{2}\right) \omega_{1}^{2}+2 \omega_{1}^{4}\right)$

$n^{4}=-\left(4 e f-g^{2}+2 e n^{2}+2 f n^{2}-\left(-2 e-2 f+2 n^{2}\right) \omega^{2}+\omega^{4}\right)$

$\left(\omega_{1}^{2}+\omega_{2}^{2}\right)=-2\left(e+f-n^{2}\right),\left(\omega_{1}^{2} \omega_{2}^{2}\right)=4 e f-g^{2}+2 e n^{2}+2 f n^{2}+n^{4}$

$4 e f-g^{2}+2 e n^{2}+2 f n^{2}+n^{4}+2\left(e+f-n^{2}\right) \omega^{2}+\omega^{4}=0$

$\left(e+f-n^{2}\right) \omega^{2}=-\frac{\left(4 e f-g^{2}+2 e n^{2}+2 f n^{2}+n^{4}+\omega^{4}\right)}{2}$

Simplify 


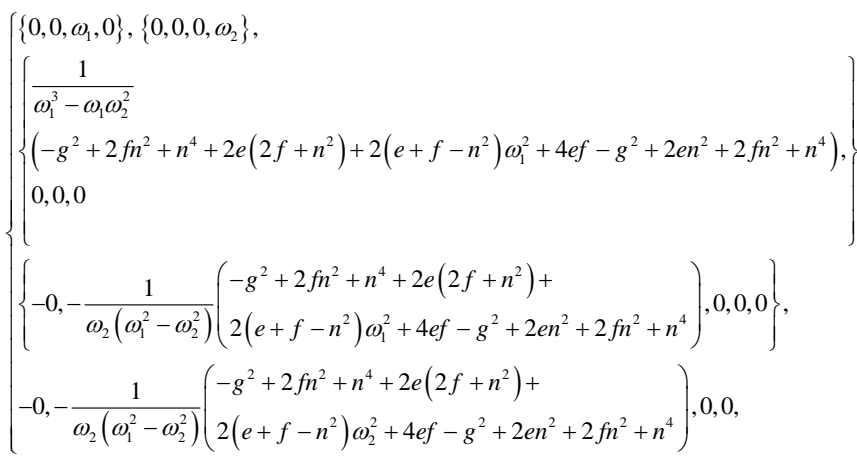

Simplify

cinvMc =

$\left\{\left\{0,0, \omega_{1}, 0\right\},\left\{0,0,0, \omega_{2}\right\},\left\{\frac{\omega_{1}}{\omega_{1}^{2}-\omega_{2}^{2}}, 0,0,0,\right\}\left\{0, \frac{\omega_{2}}{-\omega_{1}^{2}+\omega_{2}^{2}}, 0,0\right\}\right\}$

$H_{2}=\frac{1}{2}\left\{P_{1}, P_{2}, Q_{1}, Q_{2}\right\} \cdot \operatorname{cin} v M c \quad \cdot\left\{P_{1}, P_{2}, Q_{1}, Q_{2}\right\}$

$=\frac{1}{2}\left(P_{1} Q_{1} \omega_{1}+P_{2} Q_{2} \omega_{2}+\frac{P_{1} Q_{1} \omega_{1}}{\omega_{1}^{2}-\omega_{2}^{2}}+\frac{P_{2} Q_{2} \omega_{2}}{-\omega_{1}^{2}+\omega_{2}^{2}}\right)$

Expand

$\frac{P_{1} Q_{1} \omega_{1}}{2\left(\omega_{1}^{2}-\omega_{2}^{2}\right)}+\frac{P_{1} Q_{1} \omega_{1}^{3}}{2\left(\omega_{1}^{2}-\omega_{2}^{2}\right)}-\frac{P_{2} Q_{2} \omega_{2}}{2\left(\omega_{1}^{2}-\omega_{2}^{2}\right)}+\frac{P_{2} Q_{2} \omega_{1}^{2} \omega_{2}}{2\left(\omega_{1}^{2}-\omega_{2}^{2}\right)}-\frac{P_{1} Q_{1} \omega_{1} \omega_{2}^{2}}{2\left(\omega_{1}^{2}-\omega_{2}^{2}\right)}-$
$\frac{P_{2} Q_{2} \omega_{2}^{3}}{2\left(\omega_{1}^{2}-\omega_{2}^{2}\right)}$

Simplify

$\frac{P_{2} Q_{2} \omega_{2}\left(-1+\omega_{1}^{2}-\omega_{2}^{2}\right)+P_{1} Q_{1} \omega_{1}\left(1+\omega_{1}^{2}-\omega_{2}^{2}\right)}{2\left(\omega_{1}^{2}-\omega_{2}^{2}\right)}$
$=\frac{1}{2}\left\{P_{1}, P_{2}, Q_{1}, Q_{2}\right\}$.

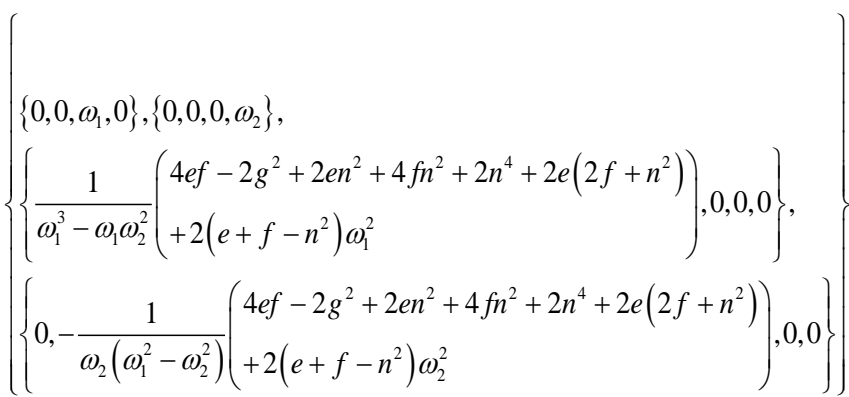

$\left\{P_{1}, P_{2}, Q_{1}, Q_{2}\right\}$

$=\frac{1}{2}$

$\left\{\begin{array}{l}P_{1} Q_{1} \omega_{1}+P_{2} Q_{2} \omega_{2}-\frac{1}{\omega_{2}\left(\omega_{1}^{2}-\omega_{2}^{2}\right)} \\ \left(P_{2} Q_{2}\left(4 e f-2 g^{2}+2 e^{2}+4 \mathrm{fn}^{2}+2 n^{4}+2 e\left(2 f+n^{2}\right)+2\left(e+f-n^{2}\right) \omega_{2}^{2}\right)\right)+ \\ \frac{1}{\omega_{1}^{3}-\omega_{1} \omega_{2}^{2}} \\ \left(P_{1} Q_{1}\left(4 e f-2 g^{2}+2 e n^{2}+4 \mathrm{fn}^{2}+2 n^{4}+2 e\left(2 f+n^{2}\right)+2\left(e+f-n^{2}\right) \omega_{2}^{2}\right)\right)\end{array}\right.$
Simplify

$\frac{1}{2 \omega_{1} \omega_{2}\left(\omega_{1}^{2}-\omega_{2}^{2}\right)}$
$\left(\begin{array}{l}-P_{2} Q_{2} \omega_{1}\left(2\left(-g^{2}+2 f n^{2}+n^{4}+2 e\left(2 f+n^{2}\right)\right)+\left(2\left(e+f-n^{2}\right)-\omega_{1}^{2}\right) \omega_{2}^{2}+\omega_{2}^{4}\right)+ \\ P_{1} Q_{1} \omega_{2}\left(2\left(-g^{2}+2 f n^{2}+n^{4}+2 e\left(2 f+n^{2}\right)\right)+\omega_{1}^{4}+\omega_{1}^{2}\left(2\left(e+f-n^{2}\right)-\omega_{2}^{2}\right)\right)\end{array}\right)$
since
$\left(2\left(-g^{2}+2 f n^{2}+n^{4}+2 e\left(2 f+n^{2}\right)\right)+2\left(e+f-n^{2}\right) \omega_{2}^{2}-\omega_{1}^{2} \omega_{2}^{2}+\omega_{2}^{4}\right)=-2 \omega_{1}^{2} \omega_{2}^{2}$

so above

$$
\begin{gathered}
H_{2}=\frac{1}{2 \omega_{1} \omega_{2}\left(\omega_{1}^{2}-\omega_{2}^{2}\right)}\left(-P_{2} Q_{2} \omega_{1}\left(-2 \omega_{1}^{2} \omega_{2}^{2}\right)+P_{1} Q_{1} \omega_{2}\left(-2 \omega_{1}^{2} \omega_{2}^{2}\right)\right) \\
=\frac{2 P_{2} Q_{2} \omega_{1}^{3} \omega_{2}^{2}-2 P_{1} Q_{1} \omega_{1}^{2} \omega_{2}^{3}}{2 \omega_{1} \omega_{2}\left(\omega_{1}^{2}-\omega_{2}^{2}\right)}
\end{gathered}
$$

Simplify

$$
=\frac{\omega_{1} \omega_{2}\left(P_{2} Q_{2} \omega_{1}-P_{1} Q_{1} \omega_{2}\right)}{\omega_{1}^{2}-\omega_{2}^{2}}
$$

Expand

$$
\begin{gathered}
=\frac{P_{2} Q_{2} \omega_{1}^{2} \omega_{2}}{\omega_{1}^{2}-\omega_{2}^{2}}-\frac{P_{1} Q_{1} \omega_{1} \omega_{2}^{2}}{\omega_{1}^{2}-\omega_{2}^{2}} \\
=\frac{P_{2} Q_{2} \omega_{1}^{2} \omega_{2}}{\omega_{1}^{2}-\omega_{2}^{2}}-\frac{P_{1} Q_{1} \omega_{1} \omega_{2}^{2}}{\omega_{1}^{2}-\omega_{2}^{2}} \\
P_{1}=\left(1-\frac{\omega_{1}}{\omega_{2}}\right) \alpha_{1} ; Q_{1}=\left(1+\frac{\omega_{1}}{\omega_{2}}\right) \beta_{1} ; P_{2}=\left(1-\frac{\omega_{2}}{\omega_{1}}\right) \alpha_{2} ; Q_{2}=\left(1+\frac{\omega_{2}}{\omega_{1}}\right) \beta_{2}
\end{gathered}
$$

Change the scale as above

then $H_{2}=\frac{P_{2} Q_{2} \omega_{1}^{2} \omega_{2}}{\omega_{1}^{2}-\omega_{2}^{2}}-\frac{P_{1} Q_{1} \omega_{1} \omega_{2}^{2}}{\omega_{1}^{2}-\omega_{2}^{2}}$

$\mathrm{H}_{2}=$ Simplify

$=\alpha_{1} \beta_{l} \omega_{l}+\alpha_{2} \beta_{2} \omega_{2}$

which is required normal form for given Hamiltonian

\section{References}

[1] Benettin, G.; Fasso, F.; Guzzo, M. Nekhoroshev, 1998, Stability of $\mathrm{L}_{4}$ and $\mathrm{L}_{5}$ in the spatial restricted three-body problem. Regular and $\begin{array}{lllll}\text { chaotic dynamics } & \text { V. } & \text { No. }\end{array}$ http://dx.doi.org/10.1070/rd1998v003n03ABEH000080.

[2] Celleti, A. and Giorgilli, A., 1991, on the stability of the Lagrangian points in the spatial Restricted Three Body Problem. Celest. Mech. Dynam. Astrr. 50(1): 31-58. http://dx.doi.org/10.1007/BF00048985.

[3] Duskos, C. N. and Markellos, V.V. 2006, Out-of-plane equilibrium points in the restricted three body problem with oblateness, A \& a 446, 357-360 
[4] Ito, H., 1992, Integrability of Hamiltonian systems and Birkhoff normal forms in the simple resonance case, Math. Ann. 292, 411444. http://dx.doi.org/10.1007/BF01444629.

[5] Jorba, A., 2001, Numerical computation of the normal behaviour of invariant curves of n-dimensional maps. Nonlinearity, 14(5): $943-$ 976. http://dx.doi.org/10.1088/0951-7715/14/5/303.

[6] Kusvah, B. S., Sharma J. P. and Ishwar, B. 2007, Non-linear stability in generalisedphotogravitational restricted three body problems with P-R drag. Ap\& SS, 312, 279-293. http://dx.doi.org/10.1007/s10509-007-9688-0.

[7] Meyer, K. R.; Hall, G. R., 1992, Introduction to Hamiltonian Dynamical Systems and the N-Body Problem. Springer-Verlag. New York. http://dx.doi.org/10.1007/978-1-4757-4073-8.

[8] Oberti, P. and Vienne, A., 2003, An upgraded theory for Helene, Telesto and Calypso. A\&A 397, 353-359.

[9] Poschel, J., 1982, the concept of integrability on Cantor sets for Hamiltonian systems, Celestial Mechanics 28, 133-139. http://dx.doi.org/10.1007/BF01230665.

[10] Sharma, R. K. and Subba Rao, P.V., 1976, Stationary solutions and their characteristic exponents in the restricted three-body problem when the more massive primary is an oblate spheroid. Celes. Mech., 13,137. http://dx.doi.org/10.1007/BF01232721.

[11] Sokol'skii, A. G., 1978, Proof of the stability of Lagrangian solutions for a critical mass ratio. Sov. Astronom. Lett. 79-81.

[12] Szebehely, V., 1967, Theory of orbits. Academic Press. New York. 